\title{
Association between hysterectomy wait-time and all-cause mortality for micro-invasive cervical cancer: treatment implications during the coronavirus pandemic
}

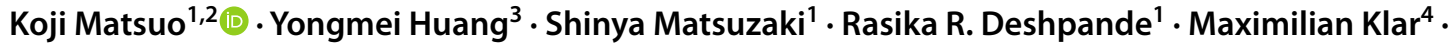 \\ Lynda D. Roman ${ }^{1,2}$. Jason D. Wright ${ }^{3}$
}

Received: 2 July 2021 / Accepted: 19 July 2021 / Published online: 23 July 2021

(c) The Author(s), under exclusive licence to Springer-Verlag GmbH Germany, part of Springer Nature 2021

\section{Dear Editor,}

In 2021, a global pandemic caused by a novel coronavirus (COVID-19) continues to be a major health threat. In the United States, nearly 33.5 million people have tested positive for COVID-19 and over 602,400 patients have died from complications related to COVID-19 as of early July 2021 [1]. The pandemic crisis is stressing the healthcare systems creating unprecedented challenges in providing timely oncologic care. Multiple studies have demonstrated that COVID19 has resulted in delayed cancer care [2,3].

A recent high-quality meta-analysis concluded that cancer treatment delay is associated with increased mortality in various malignancies, but valid data on cervical cancer remain scant [4]. Given that the majority of women with early-stage cervical cancer are treated surgically with hysterectomy, we examined the association between hysterectomy wait-time and oncologic outcomes for women with microinvasive cervical cancer.

This retrospective observational study examined women with stage IA squamous, adenocarcinoma, and adenosquamous carcinomas of the uterine cervix diagnosed from

Jason D. Wright

jw2459@columbia.edu

1 Division of Gynecologic Oncology, Department of Obstetrics and Gynecology, University of Southern California, Los Angeles, CA, USA

2 Norris Comprehensive Cancer Center, University of Southern California, Los Angeles, CA, USA

3 Division of Gynecologic Oncology, Department of Obstetrics and Gynecology, Columbia University College of Physicians and Surgeons, 161 Fort Washington Avenue, 8th Floor, New York, NY 10032, USA

4 Department of Obstetrics and Gynecology, University of Freiburg, Freiburg, Germany
2004 to 2015 in the National Cancer Database. All women underwent primary hysterectomy. Cases with no wait-time were excluded due to the assumption of occult malignancy. Associations between surgical wait-time, defined as time interval from cancer diagnosis to hysterectomy, and oncologic outcomes including surgical-pathological factors (pathological parametrical invasion, nodal metastasis, and lympho-vascular space invasion) and all-cause mortality were examined [5].

A generalized linear regression model was used to assess the association between wait-time and pathologic characteristics. Binary logistic regression and Cox proportional hazards regression models with restricted cubic spline transformation of surgery wait-time were used to assess the nonlinear associations between outcome measures, adjusting for other patient and tumor characteristics. The Columbia University Institutional Review Board deemed exempted this study due to the use of publicly available data.

A total of 2732 women were examined. The median age was 43 (IQR 36-52) years. Squamous histology $(n=1792$, $65.5 \%)$ and stage IA1 disease $(n=1185,43.4 \%)$ were the most frequent tumor characteristics. The median hysterectomy wait-time was 6 (IQR 4-9) weeks. Non-Hispanic Black and Hispanic patients, and uninsured and Medicaid insurance were independently associated with longer hysterectomy wait-time in multivariable analysis (all, $P<0.001$; Table 1). Longer hysterectomy wait-time was not associated with increased risks of pathological parametrial involvement, regional lymph node metastasis, or lympho-vascular space invasion (Fig. 1A-C). The median follow-up was 4.5 (IQR 2.2-7.2) years, and $136(5.0 \%)$ deaths occurred. Longer hysterectomy wait-time was not associated with allcause mortality risk $(P=0.431$; Fig. $1 \mathrm{D})$.

The observed result with absence of association between hysterectomy wait-time and mortality risk is somehow reassuring. Notably, our results for micro-invasive cervical 
Table 1 Multivariable model for hysterectomy wait-time in micro-invasive cervical cancer

\begin{tabular}{|c|c|c|c|}
\hline & No. $(\%)$ & Mean (SD) & $\begin{array}{l}\text { Estimated parameters } \\
\text { (beta) }(95 \% \mathrm{CI})^{\S}\end{array}$ \\
\hline No. patients & $2732(100.0)$ & & \\
\hline \multicolumn{4}{|l|}{ Age } \\
\hline$<40$ & $1018(37.3)$ & $7.2(4.9)$ & Referent \\
\hline $40-49$ & $907(33.2)$ & $7.3(4.6)$ & $0.29(-0.84,1.41)$ \\
\hline $50-59$ & $458(16.8)$ & $7.9(5.1)$ & $0.76(-0.39,1.92)$ \\
\hline $60-69$ & $260(9.5)$ & $7.9(5)$ & $0.87(-0.38,2.11)$ \\
\hline $70-79$ & $70(2.6)$ & $7.2(4.6)$ & $0.13(-1.56,1.83)$ \\
\hline$\geq 80$ & $19(0.7)$ & $5.1(2.8)$ & $-1.74(-4.17,0.68)$ \\
\hline \multicolumn{4}{|l|}{ Race/ethnicity } \\
\hline Non-Hispanic: White & $1803(66.0)$ & $6.8(4.5)$ & Referent \\
\hline Non-Hispanic: Black & $305(11.2)$ & $8.7(5.5)$ & $1.21(0.60,1.81)^{* *}$ \\
\hline Hispanic & $356(13.0)$ & $9.2(5.5)$ & $1.23(0.64,1.82)^{* *}$ \\
\hline Non-Hispanic: Other & $109(4.0)$ & $8.0(4.7)$ & $0.58(-0.34,1.50)$ \\
\hline Unknown & $159(5.8)$ & $7.2(4.9)$ & $0.49(-0.27,1.25)$ \\
\hline \multicolumn{4}{|l|}{ Insurance status } \\
\hline Not insured & $176(6.4)$ & $9.5(5.9)$ & $2.09(1.33,2.84)^{* *}$ \\
\hline Private & $1656(60.6)$ & $6.7(4.3)$ & Referent \\
\hline Medicaid & $539(19.7)$ & $8.7(5.6)$ & $1.49(1.01,1.97)^{* *}$ \\
\hline Medicare & $240(8.8)$ & $7.4(4.9)$ & $0.52(-0.28,1.32)$ \\
\hline Other Government & $44(1.6)$ & $7.2(4.5)$ & $0.53(-0.86,1.93)$ \\
\hline Unknown & $77(2.8)$ & $8.6(4.8)$ & $1.58(0.50,2.67)^{*}$ \\
\hline \multicolumn{4}{|c|}{ Neighborhood average household income } \\
\hline$<\$ 40,227$ & $583(21.3)$ & $8.3(5.2)$ & Referent \\
\hline$\$ 40,227-\$ 50,353$ & $640(23.4)$ & $7.3(4.6)$ & $-0.47(-1.03,0.09)$ \\
\hline$\$ 50,354-\$ 63,332$ & $636(23.3)$ & $7.0(4.7)$ & $-0.65(-1.26,-0.04)^{*}$ \\
\hline$\geq \$ 63,333$ & $837(30.6)$ & $7.1(4.8)$ & $-0.29(-0.99,0.41)$ \\
\hline Not available & $36(1.3)$ & $8.9(5.5)$ & $3.92(-0.22,8.05)$ \\
\hline \multicolumn{4}{|c|}{ Neighborhood education level } \\
\hline$\leq 17.6 \%$ & $702(25.7)$ & $8.4(5.3)$ & Referent \\
\hline $10.9 \%-17.5 \%$ & $753(27.6)$ & $7.4(4.8)$ & $-0.12(-0.65,0.41)$ \\
\hline $6.3 \%-10.8 \%$ & $708(25.9)$ & $6.8(4.5)$ & $-0.48(-1.10,0.13)$ \\
\hline$<6.3 \%$ & $538(19.7)$ & $6.7(4.5)$ & $-0.66(-1.38,0.07)$ \\
\hline Not available & $31(1.1)$ & $8.7(5.4)$ & $-4.20(-8.67,0.28)$ \\
\hline \multicolumn{4}{|l|}{ Urban/Rural } \\
\hline Metropolitan & $2,227(81.5)$ & $7.5(4.9)$ & Referent \\
\hline Urban & $384(14.1)$ & $6.8(4.3)$ & $-0.59(-1.14,-0.05)^{*}$ \\
\hline Rural & $46(1.7)$ & $6.3(4.6)$ & $-0.87(-2.25,0.50)$ \\
\hline Unknown & $75(2.7)$ & $8.5(5.6)$ & $0.99(-0.12,2.11)$ \\
\hline \multicolumn{4}{|c|}{ Charlson/Deyo comorbidity } \\
\hline 0 & $2,397(87.7)$ & $7.4(4.8)$ & Referent \\
\hline 1 & $285(10.4)$ & $7.4(4.8)$ & $-0.37(-0.95,0.21)$ \\
\hline 2 & $50(1.8)$ & $8.1(5.6)$ & $-0.02(-1.35,1.31)$ \\
\hline \multicolumn{4}{|l|}{ Year of diagnosis } \\
\hline 2004 & $139(5.1)$ & $7.8(5.3)$ & Referent \\
\hline 2005 & $170(6.2)$ & $7.8(5.3)$ & $0.14(-0.91,1.18)$ \\
\hline 2006 & $177(6.5)$ & $7.1(4.6)$ & $-0.52(-1.56,0.51)$ \\
\hline 2007 & $182(6.7)$ & $7.5(4.9)$ & $-0.13(-1.16,0.89)$ \\
\hline 2008 & $224(8.2)$ & $7.2(4.7)$ & $-0.33(-1.32,0.65)$ \\
\hline 2009 & $284(10.4)$ & $7.7(4.7)$ & $0.08(-0.87,1.02)$ \\
\hline 2010 & $261(9.6)$ & $7.0(4.7)$ & $-0.60(-1.57,0.37)$ \\
\hline
\end{tabular}


Table 1 (continued)

\begin{tabular}{|c|c|c|c|}
\hline & No. $(\%)$ & Mean (SD) & $\begin{array}{l}\text { Estimated parameters } \\
\text { (beta) }(95 \% \mathrm{CI})^{\S}\end{array}$ \\
\hline 2011 & $251(9.2)$ & $7.3(4.8)$ & $-0.27(-1.24,0.71)$ \\
\hline 2012 & $238(8.7)$ & $7.6(5.2)$ & $-0.16(-1.14,0.83)$ \\
\hline 2013 & $256(9.4)$ & $7.3(4.7)$ & $-0.33(-1.30,0.65)$ \\
\hline 2014 & $286(10.5)$ & $7.1(4.7)$ & $-0.49(-1.44,0.47)$ \\
\hline 2015 & $264(9.7)$ & $7.4(4.9)$ & $-0.11(-1.08,0.87)$ \\
\hline \multicolumn{4}{|l|}{ Histology } \\
\hline Squamous cell & $1,792(65.6)$ & $7.6(5)$ & Referent \\
\hline Adenocarcinoma & $873(32.0)$ & $6.9(4.5)$ & $-0.23(-0.63,0.18)$ \\
\hline Adenosquamous & $67(2.5)$ & $7.0(5.4)$ & $-0.36(-1.51,0.78)$ \\
\hline \multicolumn{4}{|l|}{ Clinical Stage IA } \\
\hline IA 1 & $1185(43.4)$ & $7.4(4.8)$ & Referent \\
\hline IA2 & $449(16.4)$ & $7.4(4.9)$ & $0.16(-0.35,0.68)$ \\
\hline IA NOS & $1098(40.2)$ & $7.3(4.9)$ & $0.01(-0.39,0.41)$ \\
\hline \multicolumn{4}{|l|}{ Grade } \\
\hline Well & $595(21.8)$ & $7.4(4.8)$ & Referent \\
\hline Moderate & $822(30.1)$ & $7.3(4.7)$ & $-0.44(-0.94,0.07)$ \\
\hline Poorly & $323(11.8)$ & $7.2(4.9)$ & $-0.64(-1.29,0.01)$ \\
\hline Unknown & $992(36.3)$ & $7.6(5)$ & $-0.20(-0.68,0.29)$ \\
\hline \multicolumn{4}{|l|}{ Facility location } \\
\hline Eastern & 344 (12.6) & $7.8(4.9)$ & Referent \\
\hline South & $449(16.4)$ & $7.0(4.4)$ & $-0.64(-1.31,0.04)$ \\
\hline Midwest & $647(23.7)$ & $7.1(4.6)$ & $-0.67(-1.30,-0.05)^{*}$ \\
\hline West & $274(10.0)$ & $8.8(5.5)$ & $0.75(-0.01,1.50)$ \\
\hline Unknown & $1018(37.3)$ & $7.2(4.9)$ & Non-estimated \\
\hline \multicolumn{4}{|l|}{ Facility type } \\
\hline Community cancer program & $88(3.2)$ & $7.6(4.2)$ & Referent \\
\hline Comprehensive community cancer program & $582(21.3)$ & $6.7(4.5)$ & $-0.31(-1.36,0.75)$ \\
\hline Academic/research program & 863 (31.6) & $8.2(5.1)$ & $0.63(-0.40,1.65)$ \\
\hline Integrated network cancer program & $181(6.6)$ & $6.7(4.1)$ & $-0.27(-1.47,0.93)$ \\
\hline Other or unknown & $1018(37.3)$ & $7.2(4.9)$ & Non-estimated \\
\hline
\end{tabular}

No number; SD, standard deviation; $C I$ confidence interval; and NOS not otherwise specified

Mean wait-time (weeks) from cervical cancer diagnosis to hysterectomy is shown

${ }^{\S}$ Estimated parameters (beta) from generalized linear regression model. $* P<0.05, * * P<0.001$. Due to the collinearity between age $<40$, facility location and type unknown categories, betas were non-estimated for facility location and type unknown categories

cancer differ from data for stage IB tumors in which longer wait times to hysterectomy are associated with increased mortality [5]. Our findings may be due, at least in part, to the favorable prognosis for micro-invasive cervical cancer. Important limitations in this study included missing information on underlying reason of hysterectomy delay, comorbidities, occult cancer diagnosis, and use of excisional biopsy prior to hysterectomy.
As there are few data to describe the survival effect of delay in hysterectomy in cervical cancer $[4,6]$, our analysis of stage IA tumors provides valuable information in the management of women with early-stage cervical cancer and suggests that recommendations by recent expert panels to postpone hysterectomy for $6-8$ weeks among patients with early-stage cervical cancer in centers or regions with a high burden of COVID-19 disease are reasonable for stage IA disease and do not adversely impact survival [7]. 


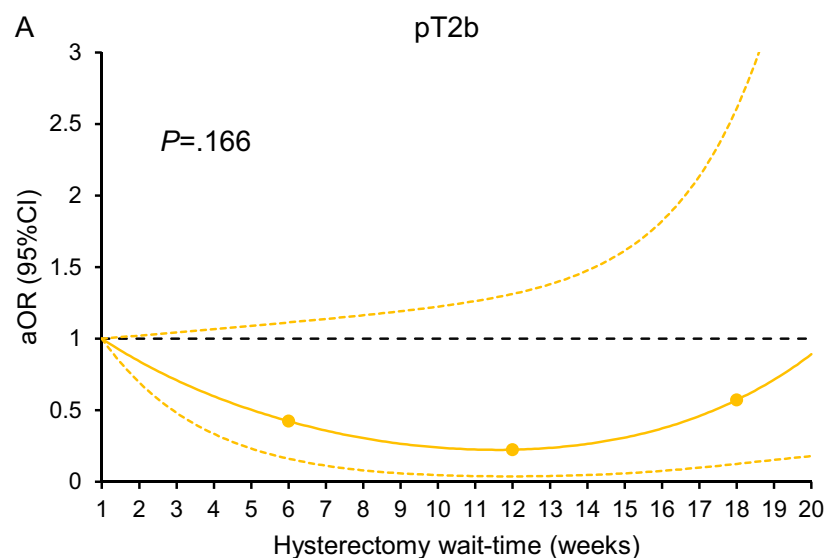

C

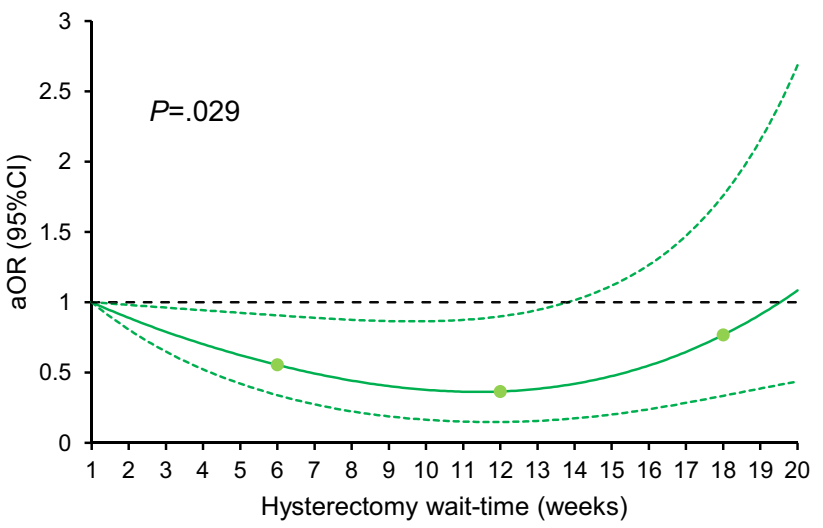

Fig. 1 Associations between hysterectomy wait-time and oncologic outcomes and all-cause mortality (adjusted model). A total of 2,732 women with clinical stage IA cervical cancer who had primary hysterectomy were examined. Adjusted-odds ratio for pathological stage T2b (A), LVSI (B), and nodal metastasis (C), and adjustedhazard ratio for all-cause mortality (D) are shown by week of hysterectomy wait-time. Waiting time was coded using restricted cubic spline transformation with clinically relevant cut-points at 6,12 , and 18 weeks. The Y-axis represents the effect size (adjusted-odds ratio or adjusted-hazard ratio). The $\mathrm{X}$-axis represents the wait-time (week) from cervical cancer diagnosis to surgical treatment with hysterectomy. Week 1 is set as the reference. The solid line represents the esti-

Author contributions KM: Conceptualization, funding acquisition, investigation, methodology, project administration, writing (original draft). YH: Investigation, data collection, analysis, validation, manuscript editing. SM: Investigation, interpretation, manuscript reviewing and editing. RR Deshpande: Investigation, interpretation, manuscript reviewing and editing. MK: Investigation, interpretation, manuscript reviewing and editing. LDR: Investigation, interpretation, manuscript reviewing and editing. JDW: Conceptualization, investigation, methodology, project administration, manuscript supervision, reviewing editing.

Funding Ensign Endowment for Gynecologic Cancer Research (K.M.). The funder had no role in the design and conduct of the study; collection, management, analysis, and interpretation of the data; preparation,
B

LVSI

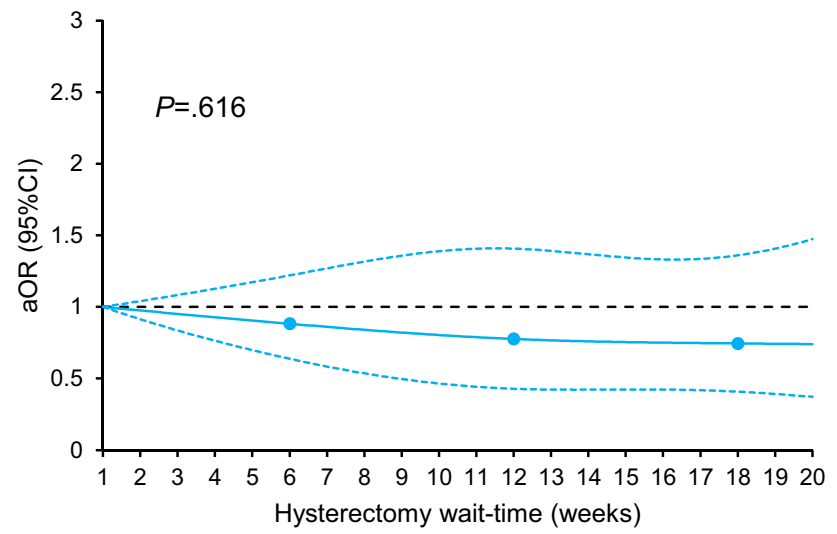

D

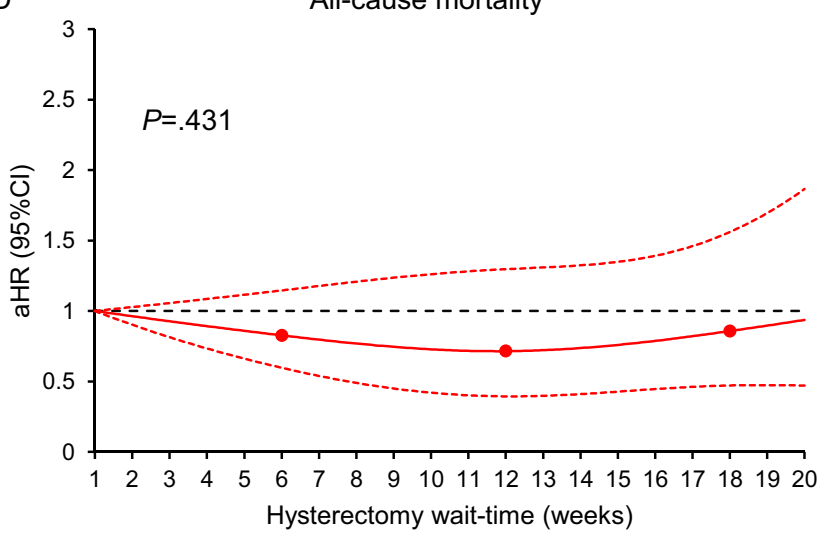

mate as adjusted-effect size. The dashed lines are corresponding 95\% confidence interval. Three dots represent the knots. $P$ values indicate the overall associations. For the surgical-pathological factors, adjusting factors were age, year, race/ethnicity, insurance status, average neighborhood household income, average neighborhood education level, year of diagnosis, comorbidity score, urban/rural type, histology type, tumor differentiation, stage, and hospital factors (location and setting). For all-cause mortality, lympho-vascular space invasion, pathological parametrial tumor involvement, and lymph node metastasis were additionally included as covariates in the multivariable Cox proportional hazard regression model

review, or approval of the manuscript; and decision to submit the manuscript for publication.

Data Availability The data on which this study is based are publicly available upon request at https://www.facs.org/quality-programs/cancer/ncdb.

\section{Declarations}

Conflict of interest The followings are outside this work: Consultant, Clovis Oncology, and research funding, Merck, royalties, UpToDate (J.D.W.); honorarium, Chugai, textbook editorial expense, Springer, investigator meeting attendance expense, VBL Therapeutics (K.M.); none for others. 
Transparency The manuscript's corresponding author (J.D.W.) affirms that the manuscript is an honest, accurate, and transparent account of the study being reported; that no important aspects of the study have been omitted; and that any discrepancies from the study as planned (and, if relevant, registered) have been explained. National Cancer Database is a joint project of the Commission on Cancer of the American College of Surgeons and the American Cancer Society. The program is the source of the de-identified data used; and the program has not verified and is not responsible for the statistical validity of the data analysis or the conclusions derived by the study team.

\section{References}

1. United States COVID-19 Cases and Deaths by State. https:// covid.cdc.gov/covid-data-tracker/\#cases_casesper100klast7days (Accessed 7/2/2021).

2. London JW, Fazio-Eynullayeva E, Palchuk MB, Sankey P, McNair C (2020) Effects of the COVID-19 pandemic on cancer-related patient encounters. JCO Clin Cancer Inform 4:657-665
3. Jazieh AR, Akbulut H, Curigliano G et al (2020) Impact of the COVID-19 pandemic on cancer care: a global collaborative study. JCO Glob Oncol 6:1428-1438

4. Hanna TP, King WD, Thibodeau S et al (2020) Mortality due to cancer treatment delay: systematic review and meta-analysis. BMJ 371:m4087

5. Matsuo K, Huang Y, Matsuzaki S, Klar M, Wright JD (2020) Effect of delay in surgical therapy for early-stage cervical cancer: an implication in the coronavirus pandemic. Eur J Cancer 139:173-176

6. Matsuo K, Novatt H, Matsuzaki S et al (2020) Wait-time for hysterectomy and survival of women with early-stage cervical cancer: a clinical implication during the coronavirus pandemic. Gynecol Oncol 158:37-43

7. Ramirez PT, Chiva L, Eriksson AGZ et al (2020) COVID-19 global pandemic: options for management of gynecologic cancers. Int J Gynecol Cancer 30:561-563

Publisher's Note Springer Nature remains neutral with regard to jurisdictional claims in published maps and institutional affiliations. 